\title{
Static and Dynamic Properties of Ferroelectric Liquid Crystals in the Vicinity of a First-Order SmA-SmC* Phase Transition
}

\author{
Roland R. Netz* and Siegfried Hess \\ Institut für Theoretische Physik der Technischen Universität Berlin, Berlin, Germany
}

Z. Naturforsch. 47 a, 536-542 (1992); received December 24, 1991

A unified theoretical description of static and dynamic properties of ferroelectric liquid crystals in terms of a Landau free energy is presented. The shear deformation tensor is used as principal order parameter. Results for the stationary case for tilt angle variation and dielectric properties are compared with experiments on a compound with a pronounced first-order $\mathrm{SmA}-\mathrm{SmC} *$ phase transition for various strengths of the applied electric field.

\section{Introduction}

Ferroelectric liquid crystals with a rather large spontaneous electric polarization in the smectic $\mathrm{C}^{*}$ phase [1] show a pronounced first-order phase transition SmA-SmC* [1, 2]. It is the purpose of this article to present a unified theoretical description of static and dynamic properties of both phases. In particular the effect of an electric field on the first-order phase transition [3-5], the electroclinic effect [6], and the dielectric response [4-6] are treated. The aproach is somewhat analogous to the previously developed unified theory for the isotropic and nematic phases [7-9]. The specific applications considered here are restricted to the SmA and the nontwisted $\mathrm{SmC}^{*}$ states where the dynamic behavior is associated with the "soft mode" $[5,6,10]$.

This article is organized as follows. In Sect. 2, the shear deformation tensor is introduced as an order parameter distinguishing the $\mathrm{SmC}^{(*)}$ phase from the SmA phase. For a special geometry it suffices to specify a scalar shear parameter. Relaxation equations for the shear parameter and for the electric polarization are formulated in Section 3; rescaled variables are introduced. The shear deformation as a function of an applied electric field and the influence of the field on the phase transition $\mathrm{SmA}-\mathrm{SmC}^{*}$ are studied theoretically in Sect. 4 and compared with experiments. In Sect. 5, the dielectric properties associated with the shear deformation are discussed. A remark on the

\footnotetext{
* Present address: IFF, Forschungszentrum Juelich, 5170 Juelich.

Reprint requests to Prof. Dr. S. Hess, Institut für Theoretische Physik, Technische Universität Berlin, PN 7-1, Hardenbergstr. 36, W-1000 Berlin 12.
}

microscopic interpretation of the electroclinic effect is made in Section 6.

\section{Order Parameter}

The standard "order parameter" used to distinguish the smectic A and C phases is the tilt angle between the director $\boldsymbol{n}$ and the normal of the smectic layers. Instead of a rotation of the molecules and hence of $\boldsymbol{n}$ by the angle $\vartheta$, here the transition A to $C$ is envisaged as a shear deformation $[11,12]$ with fixed direction of $\boldsymbol{n}$ as indicated in Fig. 1 (a microscopic theory for the $\mathrm{SmC}$ phase using the displacement of molecules along the long molecular axis as principal degree of freedom is developed in [13]). The molecules are displaced in the direction of $\boldsymbol{n}$ by the vector $\boldsymbol{u}$ which increases
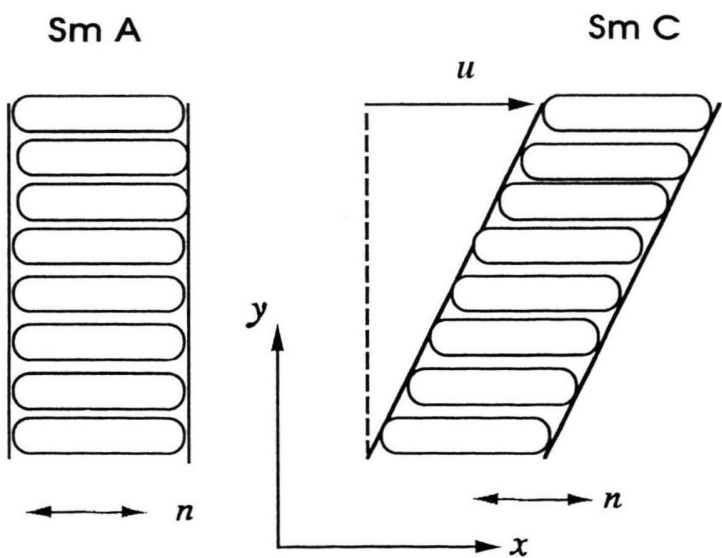

Fig. 1. Schematic description of the transition from the SmA phase to the SmC phase in terms of a shear deformation. Molecules are displaced parallel to the molecular director $n$ by the vector $\boldsymbol{u}$, which increases linearly in a direction parallel to $y$.

0932-0784 / 92 / 0300-0536 \$01.30/0. - Please order a reprint rather than making your own copy. 
linearly in a direction parallel to the unit vector $\boldsymbol{m}$ which is perpendicular to $\boldsymbol{n}$. More specifically, the position $\boldsymbol{r}^{\prime}$ of a molecule in the $\mathrm{C}$ phase is related to its position $r$ in the A phase by

$$
r^{\prime}=r+u(r)=r+r \cdot s,
$$

where

$$
\mathbf{s}=\boldsymbol{\nabla} \boldsymbol{u}=\gamma \boldsymbol{m} \boldsymbol{n}, \quad \boldsymbol{m} \perp \boldsymbol{n}
$$

is the (nonsymmetric) deformation tensor. Of course, displacements are to be measured modulo a molecular length or a layer thickness. The shear parameter $\gamma$ is related to the tilt angle $\vartheta$ by

$$
\tan \vartheta=\gamma .
$$

With $\boldsymbol{n}$ and $\boldsymbol{m}$ parallel to the $x$ - and $y$-directions, respectively, as in Fig. 1, one has

$$
\gamma=\frac{\partial u_{x}}{\partial y} .
$$

As any second rank tensor, $\mathbf{s}$ can be decomposed into its irreducible parts. Here these are the symmetric traceless part

$$
\boldsymbol{\gamma}=\overleftrightarrow{\nabla \boldsymbol{u}}=\gamma \overleftrightarrow{\boldsymbol{m} \boldsymbol{n}}
$$

and the (axial) vector

$$
\boldsymbol{\nabla} \times \boldsymbol{u}=\frac{1}{2} \gamma \boldsymbol{m} \times \boldsymbol{n}=-\frac{1}{2} \gamma \boldsymbol{l}
$$

associated with its antisymmetric part. The vectors $\boldsymbol{n}$, $\boldsymbol{m}$, and

$$
\boldsymbol{l}=\boldsymbol{n} \times \boldsymbol{m}
$$

are three mutually orthogonal unit vectors analogous to the $x, y, z$-directions of a right-handed coordinate system. In (5), $\longleftrightarrow$ refers to the symmetric traceless part of a tensor, e.g.

$$
\overleftrightarrow{a b}=\frac{1}{2}(a b+b a)-\frac{1}{3} a \cdot b \delta
$$

for the dyadic constructed from the components of two vectors $\boldsymbol{a}$ and $\boldsymbol{b} ; \boldsymbol{\delta}$ is the unit tensor.

For the geometry used in Fig. 1, the deformation tensor $\mathbf{s}$ and its decomposition into the symmetric and the antisymmetric parts read in matrix notation:

$$
\begin{aligned}
\mathbf{s} & =\gamma\left(\begin{array}{lll}
0 & 0 & 0 \\
1 & 0 & 0 \\
0 & 0 & 0
\end{array}\right) \\
& =\frac{1}{2} \gamma\left(\begin{array}{lll}
0 & 1 & 0 \\
1 & 0 & 0 \\
0 & 0 & 0
\end{array}\right)-\frac{1}{2} \gamma\left(\begin{array}{rrr}
0 & 1 & 0 \\
-1 & 0 & 0 \\
0 & 0 & 0
\end{array}\right) .
\end{aligned}
$$

(9) where $\varrho$ is the density of the fluid. The (dimensionless)
In the following, the deformation tensor $\mathbf{s}$ and the shear parameter $\gamma$ are used in the theoretical description of the $\mathrm{A}-\mathrm{C}^{*}$ transition. It seems worth pointing out that a similar approach can be used for the transition between a "straight" and a "tilted" phase in higher ordered smectics [14] as well as in columnar phases of discotic liquid crystals.

\section{Free Energy Density, Relaxation Equations}

\subsection{Free Energy Density}

The following considerations are based on a free energy density

$$
g=g^{\gamma}+g^{P}+g^{\gamma P},
$$

where $g^{\gamma}$, to be specified later, is the Landau potential associated with a deformation characterized by the shear parameter $\gamma$, and

$$
g^{\boldsymbol{P}}=-\boldsymbol{E} \cdot \boldsymbol{P}+\frac{1}{2}\left(\varepsilon_{0} \chi_{0}\right)^{-1} \boldsymbol{P} \cdot \boldsymbol{P}
$$

is the free energy density of a fluid with an electric polarization $\boldsymbol{P}$ in the presence of an electric field $\boldsymbol{E}$. In (11), $\varepsilon_{0}$ is the dielectric permeactivity of the vacuum and $\chi_{0}$ is the electric susceptibility of a nonchiral fluid or of a racemic mixture. This equation is set up in a way to give, after minimization with respect to $\boldsymbol{P}$, the well-known Debye equation for dielectric relaxation (for an introduction into this subject see [15]).

The contribution

$$
g^{\gamma P}=-E^{\text {ch }}(\nabla \times \boldsymbol{u}) \cdot \boldsymbol{P}=-E^{\text {ch }} \gamma \boldsymbol{l} \cdot \boldsymbol{P}
$$

characterizes the coupling between the electric polarization $\boldsymbol{P}$ and the deformation; for $\boldsymbol{l}$ see (7). Notice that only the component $\boldsymbol{P}^{\perp}$ of $\boldsymbol{P}$ which is perpendicular to the director $\boldsymbol{n}$ contributes in (12). In the following $\boldsymbol{n} \cdot \boldsymbol{P}=0$ is assumed, then $\boldsymbol{P}=\boldsymbol{P}^{\perp}$. Due to parity arguments, the coefficient $E^{\text {ch }}$ with the dimension of an electric field must be a pseudoscalar, i.,e. it can only be nonzero in a chiral medium, and it is written as

$$
E^{\mathrm{ch}}=\left(\chi_{\mathrm{r}}-\chi_{\mathrm{l}}\right) E_{\mathrm{r}}^{\mathrm{ch}} .
$$

$E_{\mathrm{r}}^{\mathrm{ch}}$ is the chirality coefficient of a pure right handed fluid; $\chi_{\mathrm{r}}$ and $\chi_{1}$ are the mole fractions of right and left handed molecules in a mixture. The racemic mixture corresponds to $\chi_{\mathrm{r}}=\chi_{1}$ and $E^{\mathrm{ch}}=0$.

The quantity $g^{\gamma}$ occurring in (10) is written as

$$
g^{\gamma}=\varrho k_{\mathrm{B}} T \Phi^{\gamma},
$$

Landau potential $\Phi^{\gamma}$ depends on the scalar invariants 
constructed from $\gamma$, the symmetric traceless part of the deformation tensor $\nabla \boldsymbol{u}$. Since $\operatorname{tr}(\boldsymbol{\gamma} \cdot \boldsymbol{\gamma} \cdot \boldsymbol{\gamma})=0$ for the specified deformation considered here, $\Phi^{\gamma}$ is a function of

$$
\gamma^{2}=2 \operatorname{tr}(\gamma \cdot \gamma) .
$$

Here the ansatz

$$
\Phi^{\gamma}=\frac{1}{2} A \gamma^{2}-\frac{1}{4} B \gamma^{4}+\frac{1}{6} C \gamma^{6}
$$

is used with

$$
A=A_{0}\left(1-\frac{T^{*}}{T}\right)
$$

The (positive) coefficients $A_{0}, B, C$ are assumed to be practically independent of the temperature $T$ in the vicinity of the pseudo-critical temperature $T^{*}$. Note that (16) has been chosen such that the SmA-SmC transition is of first order. With $\gamma=\tan \vartheta$ approximated by the tilt angle $\vartheta$, (16) corresponds to the ansatz used in [16-19]. A model taking into account a spatial rotation of the tilt vector and, in addition, a biquadratic coupling between polarization and tilt angle has been worked out in [20]. However, although the functional form of (16) is identical to previous calculations, the non-linear relation (3) leads to differences when the tilt angle variation is compared to experiments. The divergence of the shear parameter $\gamma$ for $\vartheta \rightarrow \pi / 2$ reflects the singular character of the corresponding deformation.

\subsection{Relaxation Equations}

The change of the specific entropy $s$, associated with the time change of $\gamma$ and of $\boldsymbol{P}$ is given by

$$
T \frac{\delta s}{\delta t}=-\frac{\partial g}{\partial \gamma} \dot{\gamma}-\frac{\partial g}{\partial \boldsymbol{P}} \dot{\boldsymbol{P}}
$$

The relaxation equations

$$
\dot{\gamma}+\tau_{\gamma}^{-1}\left(\varrho k_{\mathrm{B}} T\right)^{-1} \frac{\partial g}{\partial \gamma}=0
$$

and

$$
\dot{\boldsymbol{P}}+\tau_{\boldsymbol{P}}^{-1} \varepsilon_{0} \chi_{0} \frac{\partial g}{\partial \boldsymbol{P}}=0
$$

with the relaxation times $\tau_{\gamma}$ and $\tau_{\boldsymbol{P}}$ are constitutive equations governing the time dependence of $\gamma$ and $\boldsymbol{P}$. Equation (20) describes a Debye type relaxation. Positive entropy production requires $\tau_{\gamma}>0$ and $\tau_{P}>0$ for these phenomenological coefficients. Couplings with other nonequilibrium processes, e.g. with a viscous flow are disregarded in (19), (20). Use of (10) with (11), (12), (14), (16) yields

$\dot{\gamma}+\tau_{\gamma}^{-1}\left(\frac{\partial \Phi^{\gamma}}{\partial \gamma}-\left(\varrho k_{\mathrm{B}} T\right)^{-1} E^{\mathrm{ch}} \boldsymbol{l} \cdot \boldsymbol{P}\right)=0$,

and

$\dot{\boldsymbol{P}}+\tau_{\boldsymbol{P}}^{-1} \varepsilon_{0} \chi_{0}\left(-\boldsymbol{E}+\left(\varepsilon_{0} \chi_{0}\right)^{-1} \boldsymbol{P}-E^{\mathrm{ch}} \gamma \boldsymbol{l}\right)=0$.

In the following it is assumed that $\tau_{P} \ll \tau_{\gamma}$. This corresponds to a much faster relaxation of $\boldsymbol{P}$ than of $\gamma$. Then one has

$$
\boldsymbol{P}=\varepsilon_{0} \chi_{0}\left(\boldsymbol{E}+E^{\mathrm{ch}} \gamma \boldsymbol{l}\right) .
$$

Insertion of (23) into (21) and use of (16) yields

$\dot{\gamma}+\tau_{\gamma}^{-1}\left(A_{1} \gamma-B \gamma^{3}+C \gamma^{5}-\frac{\varepsilon_{0} \chi_{0}}{\varrho k_{\mathrm{B}} T} E^{\mathrm{ch}} \boldsymbol{l} \cdot \boldsymbol{E}\right)=0$

with

$$
A_{1}=A-\frac{\varepsilon_{0} \chi_{0}}{\varrho k_{\mathrm{B}} T}\left(E^{\mathrm{ch}}\right)^{2}=A_{0}\left(1-\frac{T^{+}}{T}\right),
$$

where the new pseudo-critical temperature $T^{+}$is related to $T^{*}$ occurring in (17) by

$$
k_{\mathrm{B}} T^{+}=k_{\mathrm{B}} T^{*}+\frac{\varepsilon_{0} \chi_{0}}{\varrho A_{0}}\left(E^{\mathrm{ch}}\right)^{2} .
$$

Due to (13), equation (26) explains the observed dependence of the $\mathrm{SmA}-\mathrm{SmC}^{*}$ transition temperature on the relative concentration of right and left handed molecules in a mixture [16].

\subsection{Rescaled Variables}

For a stationary situation $(\dot{\gamma}=0)$ and in the absence of an applied electric field $(\boldsymbol{E}=0)$, (24) has solutions with $\gamma=0$ corresponding to the SmA phase and solutions with $\gamma \neq 0$ which correspond to the SmC* phase. The coexistence of both phases occurs at the temperature $T_{\mathrm{s}}$ where one has $g=0$ and $\partial g / \partial \gamma=0$; there $A_{1}$ is equal to

$$
A_{\mathrm{s}}=A_{0}\left(1-\frac{T^{+}}{T_{\mathrm{s}}}\right)=\frac{3}{16} \frac{B^{2}}{C} .
$$

At this transition temperature, the magnitude $\gamma_{\mathrm{s}}$ of $\gamma$ is determined by

$$
\gamma_{\mathrm{s}}^{2}=\frac{3}{4} \frac{B}{C} .
$$


With the help of the rescaled shear parameter $\Gamma$ defined by

$$
\gamma=\Gamma \gamma_{\mathrm{s}},
$$

the relaxation equation (24) can be written as

$$
\dot{\Gamma}+\left(a \Gamma-4 \Gamma^{3}+3 \Gamma^{5}-F\right)=0 .
$$

Here it is understood that $\dot{\Gamma}$ means a time derivative where the time is expressed in units of the reference time

$$
\tau_{\mathrm{s}}=\tau_{\gamma}\left(A_{\mathrm{s}} \gamma_{\mathrm{s}}\right)^{-1}
$$

The quantities $a$ and $F$ are given by

$$
a=A_{1} A_{\mathrm{s}}^{-1}=\left(1-\frac{T^{+}}{T}\right)\left(1-\frac{T^{+}}{T_{\mathrm{s}}}\right)^{-1}
$$

and

$$
F=\frac{\varepsilon_{0} \chi_{0}}{\varrho k_{\mathrm{B}} T \gamma_{\mathrm{s}} A_{\mathrm{s}}} E^{\mathrm{ch}} \boldsymbol{l} \cdot \boldsymbol{E} .
$$

Comparison of (30) with (24) shows that the coefficients $B$ and $C$ are now replaced by simple numbers. Thus just two control parameters occur in (30), viz. $a$, which is determined by the temperature and $F$, which is a measure for the strength of the external field.

Before applications of (30) are discussed, it is mentioned that the electric polarization $\boldsymbol{P}^{\gamma}$ associated with the shear parameter $\gamma$, cf. (23), is given by

$$
\boldsymbol{P}^{\gamma}=\varepsilon_{0} \chi_{0} E^{\mathrm{ch}} \gamma_{\mathrm{s}} \Gamma \boldsymbol{l}=\boldsymbol{P}^{\mathrm{s}} \Gamma \boldsymbol{l},
$$

where $P^{\mathrm{s}}=\varepsilon_{0} \chi_{0} E^{\mathrm{ch}} \gamma_{\mathrm{s}}$ is the magnitude of the spontaneous polarization at the transition temperature $T_{\mathrm{s}}$.

\section{Deformation as Function of the Applied Electric Field}

\subsection{Influence of an Electric Field on the Phase \\ Transition}

The stationary solution of (30), e.g. written as

$$
a=4 \Gamma^{2}-3 \Gamma^{4}+\frac{F}{\Gamma},
$$

for $\Gamma \neq 0$ can be used to analyze the influence of an electric field $\boldsymbol{E}$, cf. (33), on the phase transition $\mathrm{SmA}-$ SmC*. In Fig. 2, $\Gamma$ as given by (35) is displayed as function of $a$ for various values of the field parameter $F$. It is recalled that $a=1$ and $a=0$ correspond to the transition temperature $T_{\mathrm{s}}$ and to the pseudocritical temperature $T^{+}$, cf. (25). The existence of more than

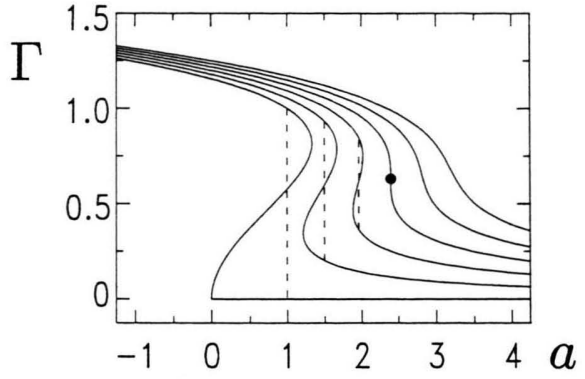

Fig. 2. Solid lines show the shear parameter $\Gamma$ as a function of the rescaled temperature $a$ as given by (35) for the following values of the rescaled electric field (from left to right): 0 , $0.27,0.54,0.81,1.08,1.35$. The broken lines indicate coexistence values of the shear parameter for field values smaller than $F_{\mathrm{c}} \approx 0.81$; the critical point as indicated by a filled cricle.

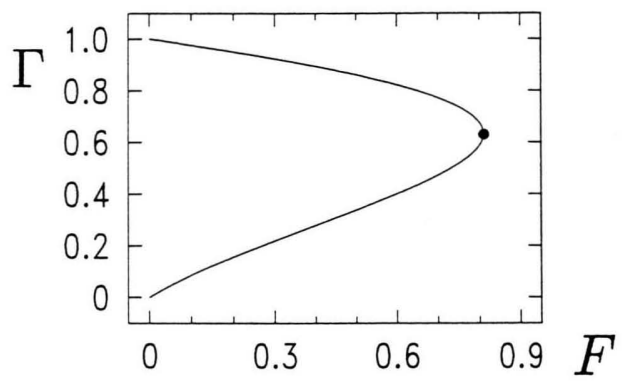

Fig. 3. Values of the shear parameter $\Gamma$ for the coexisting ordered states as a function of the rescaled electric field $F$. As $F$ approaches $F_{\mathrm{c}} \approx 0.81$, the difference of the shear parameter values between the coexisting phases goes continuously to zero.

one value of $\Gamma$ for given $a$ and $F$ corresponds to a multiphase behavior the stability of which has to be analyzed. Small deviations $\delta \Gamma$ from the stationary values will decay to zero provided that

$$
a-12 \Gamma^{2}+15 \Gamma^{4}>0 \text {. }
$$

The absolute stability of a solution $\Gamma$ of (35) for given $a$ and $F$ can be inferred from the values of the potential function

$$
G=\frac{1}{2} a \Gamma^{2}-\Gamma^{4}+\frac{1}{2} \Gamma^{6}-F \Gamma .
$$

Coexisting states correspond to two minima of $G$ of equal height. The search for these values of $\Gamma$ is analogous to the Maxwell construction and is indicated by the vertical broken lines in Figure 2. In Fig. 3, these values of $\Gamma$ for the coexisting phases are shown as function of $F$. Above a critical value $F_{\mathrm{c}}$ of the field only one equilibrium value of $\Gamma$ exists. 
The critical point occurs at

$$
\begin{aligned}
& \Gamma_{\mathrm{c}}=(2 / 5)^{1 / 2} \approx 0.63, \quad F_{\mathrm{c}}=8 \Gamma_{\mathrm{c}}^{5} \approx 0.81, \\
& a_{\mathrm{c}}=12 / 5=2.4
\end{aligned}
$$

and is indicated in Figs. 2 and 3 by a filled circle. The influence of an electric field on the phase transition $\mathrm{SmA}-\mathrm{SmC}^{*}$ and the existence of a critical point has been observed experimentally and interpreted theoretically with the help of an essentially equivalent Landau theory [21].

\subsection{Comparison with Experimental Results}

In Fig. 4, experimental data $[1,17,19]$ for the compound $\mathrm{A} 7$ of the tilt angle $\vartheta$ as function of the temperature $T$ for three values of the applied electric field $E$ corresponding to values of $F$ of $0,1.54,4.62$ are compared with the theoretical curves. The parameters needed for the quantitative comparison with the specific substance considered, viz.

$T^{+}=72.93^{\circ} \mathrm{C}, \quad T_{\mathrm{s}}=73.1^{\circ} \mathrm{C}, \quad T_{\mathrm{s}}-T^{+}=0.17^{\circ} \mathrm{C}$,

$\varrho k_{\mathrm{B}} A_{0}=8.9 \cdot 10^{4} \mathrm{~J}\left(\mathrm{~m}^{3} \mathrm{~K}\right)^{-1} \mathrm{rad}^{-2}, \quad \chi_{0}=4.2$,

$E^{\mathrm{ch}}=8.5 \cdot 10^{7} \mathrm{~J}(\mathrm{Cm} \mathrm{rad})^{-1}, \quad \vartheta_{\mathrm{s}}=14.6^{\circ}$,

were inferred from the temperature dependence of the tilt angle in the SmA phase (electroclinic effect) as given by $\Gamma=a^{-1} F$ with (29), (32), (33) and by the temperature dependence of the extra contribution to the dielectric coefficient associated with $\gamma \neq 0$ in the SmA phase as discussed in the following section.

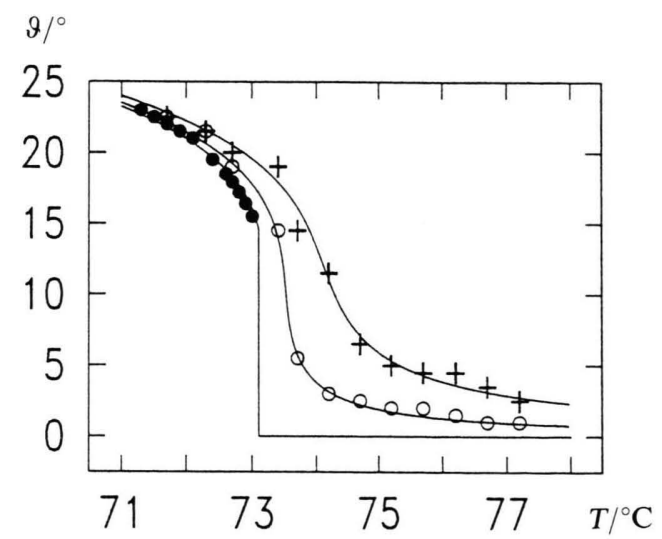

Fig. 4. Comparison of the tilt angle variation as given by (35) with experimental data for the compound A7 [17] for three values of the applied electric field corresponding to $F=0$ (filled circles), 1.54 (open circles), 4.62 (crosses). The procedure of relating experimental and theoretical quantities is described in the text.

\section{Dielectric Coefficient}

\subsection{General Remarks}

Let the applied electric field be the sum of a constant field $\boldsymbol{E}_{0}$ and a small alternating part $\delta \boldsymbol{E} \sim e^{i \omega t}$ with the frequency $\omega$. The alternating part of $\delta \boldsymbol{P}$ of the electric polarization associated with the corresponding alternating part $\delta \gamma$ of $\gamma$ is related to $\delta \boldsymbol{E}$ by $[4,5]$

$$
\delta \boldsymbol{P}=\varepsilon_{0} \Delta \varepsilon_{\perp} \delta \boldsymbol{E}
$$

with the coefficient $\Delta \varepsilon_{\perp}$, where the subscript “ $\perp$ " indicates that $\delta \boldsymbol{P}$ is perpendicular to the director $\boldsymbol{n}$. Due to (23) and (33), equation (39) is equivalent to

$$
\delta \Gamma=R \Delta \varepsilon_{\perp} \delta F
$$

with

$$
R=\varepsilon_{0} \varrho k_{\mathrm{B}} T A_{\mathrm{s}}\left(\varepsilon_{0} \chi_{0} E^{\mathrm{ch}}\right)^{-2} .
$$

Use of (30) leads to

$$
\left(-i \Omega+a-12 \Gamma_{0}^{2}+15 \Gamma_{0}^{4}\right) \delta \Gamma=\delta F
$$

with the dimensionless frequency variable

$$
\Omega=\omega \tau_{\mathrm{s}} ;
$$

for $\tau_{\mathrm{s}}$ see (31). In (42), terms nonlinear in $\delta \Gamma$ are disregarded. The stationary value $\Gamma_{0}$ of the shear parameter $\Gamma$ is to be determined from

$$
a \Gamma_{0}-4 \Gamma_{0}^{3}+3 \Gamma_{0}^{5}=F_{0},
$$

which is essentially equivalent to (35) for $\Gamma_{0} \neq 0$. The quantity $F_{0}$ is given by (33) with the electric field $\boldsymbol{E}$ replaced by the static field $\boldsymbol{E}_{0}$. Comparison of (42) with (40) yields the desired expression for $\Delta \varepsilon_{\perp}$, viz.

$$
R \Delta \varepsilon_{\perp}=\left(-i \Omega+a-12 \Gamma_{0}^{2}+15 \Gamma_{0}^{4}\right)^{-1} .
$$

Some special cases are considered next.

\subsection{Special Applications and Comparison with Experiments}

i) In the SmA phase and in the absence of a constant external field $\left(F_{0}=0\right)$ one has $\Gamma_{0}=0$, and (45) reduces to

$$
R \Delta \varepsilon_{\perp}=a^{-1}\left(-i \Omega a^{-1}+1\right)^{-1} .
$$

The factor $a^{-1} \sim\left(T-T^{+}\right)^{-1}$ describes a pretransitional increase of the dielectric coefficient $\Delta \varepsilon_{\perp}$. The dependence on the frequency $\omega$ via the variable

$$
\Omega a^{-1}=\omega \tau, \quad \tau=\tau_{\mathrm{s}} a^{-1}
$$

corresponds to a pretransitional slowing down. An effective relaxation rate $\tau^{-1}$ proportional to $\left(T-T^{+}\right)$ 
has been observed experimentally $[6,21]$. Notice that $a>0$ in the SmA phase since $T_{\mathrm{s}}>T^{+}$.

ii) In the $\mathrm{SmC}^{*}$ phase but still for $F_{0}=0,(45)$ with (44) can be written in analogy to (46) as

with

$$
R \Delta \varepsilon_{\perp}=\tilde{a}^{-1}\left(-i \Omega \tilde{a}^{-1}+1\right)^{-1} .
$$

$$
\tilde{a}=4\left(\frac{4}{3}-a+\sqrt{\frac{4}{3}\left(\frac{4}{3}-a\right)}\right) \text {. }
$$

Notice that $\tilde{a}=0$ for $a=\frac{4}{3}$ corresponding to a temperature $T$ which is larger than the transition temperature $T_{\mathrm{s}}$.

iii) For finite values of $F_{0}$, the temperature dependence of $\Delta \varepsilon_{\perp}$ as given by (45) with (44) can be analyzed graphically if one considers these equations as a parametric representation of the form $\Delta \varepsilon_{\perp}=\Delta \varepsilon_{\perp}\left(\Gamma_{0}\right)$, $a=a\left(\Gamma_{0}\right)$ (with an additional dependence on $F_{0}$ and $\Omega$ ). This procedure can be used to plot $\left(\Delta \varepsilon_{\perp}\right)^{-1}$ versus $T$ as shown in Fig. 5 for small frequencies where one uses $\Omega=0$ in (45). The curves and the experimental points correspond to three different values of the applied field $E_{0}$. For the comparison with the exprimental data, the same material parameters (cf. Sect. 4.2) have been used as for Fig. 4, hence there are no adjustable parameters. The agreement is quite satisfactory for the higher temperatures and for higher values of the static field $E_{0}$. The deviations at small values of $E_{0}$ and in particular for $E_{0}=0$ in the $\mathrm{SmC}^{*}$ phase can be caused by an additional contribution to $\Delta \varepsilon_{\perp}$ associated with an reorientational motion of the director ("Goldstone mode").

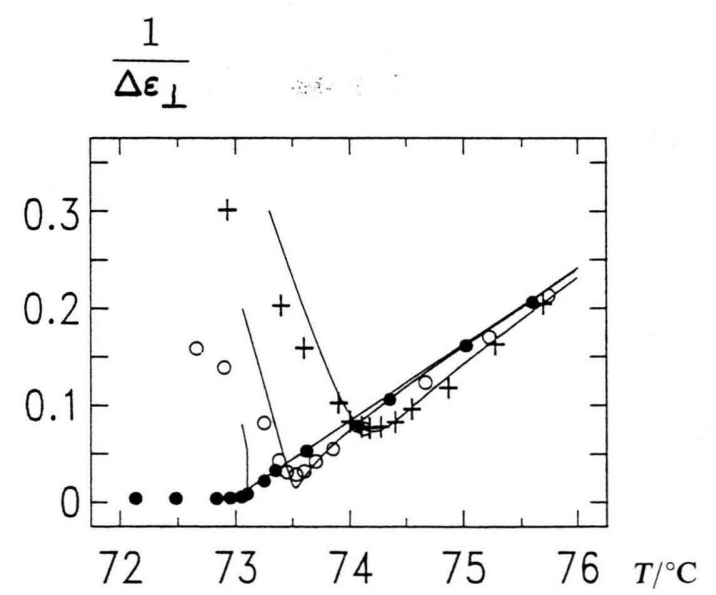

Fig. 5. Reciprocal dielectric constant as given by (44), (45) compared with data for the compound A7 [17] for values of the applied electric field corresponding to $F=0$ (filled circles), 1.54 (open circles), 4.62 (crosses).

\section{A Remark on the Electroclinic Effect}

The electroclinic effect [22], i.e. the electric-field induced rotation of the principal direction of the dielectric tensor (for optical frequencies) is usually attributed to a rotation of the director by the tilt angle $\vartheta$. It seems worth pointing out that a deformation with $\boldsymbol{n}$ fixed as discussed in Sect. 2 also gives rise to a rotation of the optical axis. This follows from the fact that the anisotropic part $\overleftrightarrow{\boldsymbol{\varepsilon}}$ of the dielectric tensor is a sum of contributions due to the molecular alignment and to the deformation (stress-optical effect) characterized by coefficients $\varepsilon_{a}$ and $\varepsilon_{b}$, respectively

$$
\overleftrightarrow{\boldsymbol{\varepsilon}}=\varepsilon_{a} \overleftrightarrow{\boldsymbol{n} \boldsymbol{n}}+\varepsilon_{b} \gamma
$$

$\boldsymbol{\gamma}$ being biaxial and defined in (5). With the director $n$ fixed and for a geometry as discussed in Sect. 2 (i.e. $\boldsymbol{\gamma}=\gamma \overleftrightarrow{\boldsymbol{m} \boldsymbol{n}}$ ), the optical axis which coincides with $\boldsymbol{n}$ for $\gamma=0$ is rotated away from $\boldsymbol{n}$ in the $\boldsymbol{n}, \boldsymbol{m}$-plane by an angle $\varphi$ given by

$$
\tan 2 \varphi=\frac{\varepsilon_{b}}{\varepsilon_{a}} \gamma
$$

For small angles $\varphi$ is proportional to $\gamma$. The comparison between experimental and theoretical results underlying Fig. 4 cannot decide about the correct microscopic interpretation of the optical tilt angle. However, the combination of an optical measurement with a scattering (x-ray or neutron) technique could provide the independent information on the molecular displacement induced by an electric field.

\section{Concluding Remarks}

A unified theoretical description of static and dynamic properties of ferroelectric liquid crystals with special emphasis on electric field effects has been presented. It has been demonstrated that the shear deformation tensor serves well as an order parameter for the $\mathrm{SmC}^{*}$ phase. By use of rescaled variables, parameters of the theory are related to quantities easily accessible to experiments, such as the transition temperature $T_{\mathrm{s}}$, the pseudo-critical temperature $T^{+}$, and the tilt angle at the transition $\gamma_{s}$. In the special stationary case, the equations describe accurately the effect of an electric field on the phase transition and the electroclinic effect, as revealed by a plot of theoretical and experimental tilt angle variation curves (see Figure 4). The dielectric response is equally well described for 
the full range of experimental data (Fig. 5), except deep in the $\mathrm{SmC}^{*}$ phase where contributions due to a reorientation of the director might be important. It is conjectured that the shear parameter $\gamma$, which is related to the tilt angle $\vartheta$ by the relation $\gamma=\tan \vartheta$, is a better starting point for an expansion in terms of a Landau free-energy expression than the tilt angle itself.

[1] Ch. Bahr and G. Heppke, Mol. Cryst. Liq. Cryst. 148, 29 (1987); 151, 69 (1987).

[2] B. R. Ratna, R. Shashidar, Geeth G. Nair, S. Krishna Prasad, Ch. Bahr, and G. Heppke, Phys. Rev. A 37, 1824 (1988).

[3] Ch. Bahr and G. Heppke, Mol. Cryst. Liq. Cryst. $\mathbf{1 5 0}$ b, 313 (1987).

[4] Ch. Bahr, G. Heppke, and N. K. Sharma, Ferroelectrics 76, 151 (1987).

[5] Ch. Bahr and G. Heppke, Phys. Rev. A 41, 4335 (1990).

[6] Ch. Bahr and G. Heppke, Liquid Crystals 2, 825 (1987).

[7] S. Hess, Z. Naturforsch. 30 a, 728, 1224 (1975); 31 a, 1507 (1976).

[8] S. Hess and I. Pardowitz, Z. Naturforsch. 36a, 554 (1981).

[9] I. Pardowitz and S. Hess, Physica 100 A, 540 (1980); J. Chem. Phys. 76, 1485 (1982).

[10] F. Gießelmann and P. Zugenmaier, Liquid Crystals 8, 361 (1990).

[11] A. J. Leadbetter, in: Molecular Physics of Liquid Crystals (G. R. Luckhurst and G. W. Gray, eds.), p. 305, Academic Press, New York 1979.

[12] P. Pieranski, E.Guyon, and P. Keller, J. Phys. Paris 36, 1005 (1975). - S. V. Yablonsky, L. M. Blinov, and S. A. Pikin, Mol. Cryst. Liq. Cryst. 127, 381 (1985).

\section{Acknowledgements}

This work has been conducted under the auspices of the Sonderforschungsbereich "Anisotrope Fluide" (Sfb 335) of the Deutsche Forschungsgemeinschaft. Financial support is gratefully acknowledged.

We thank Dr. Ch. Bahr, Prof. Dr. G. Heppke, and Prof. Dr. W. Muschik for helpful discussions.

[13] R. R. Netz and A. N. Berker, in: Phase Transitions in Liquid Crystals (S. Martellucci, ed.), Plenum Press, New York 1991

[14] Ch. Bahr and G. Heppke, Phys. Rev. A 37, 3179 (1988).

[15] S. R. De Groot and P. Mazur, in: Non-Equilibrium Thermodynamics, ch. XIV, North-Holland, Amsterdam 1963.

[16] Ch. Bahr, G. Heppke, and B. Sabaschus, Ferroelectrics 84, 103 (1988).

[17] Ch. Bahr, Dissertation, Technical University, Berlin 1988.

[18] C. C. Huang and J. M. Vimer, Phys. Rev. A 25, 3385 (1982).

[19] Ch. Bahr and G. Heppke, Ber. Bunsenges. Phys. Chem. 91, 925 (1987).

[20] T. Carlsson, B.Žekš, C. Filipič, A. Levstik, and R. Blinc, Mol. Cryst. Liq. Cryst. 163, 11 (1988).

[21] Ch. Bahr and G. Heppke, Physica A 174, 139 (1991).

[22] S. Garoff and B. Meyer, Phys. Rev. Lett. 38, 848 (1977); Phys. Rev. A 19, 338 (1979). 